\title{
Holt-Winters Forecasting Method That Takes into Account the Effect of Eid
}

\author{
Bustami, Hadi Irawansyah, M. D. H. Gamal \\ Department of Mathematics, University of Riau, Pekanbaru, Indonesia
}

Email address:

bustami_muararumbai@yahoo.co.id (Bustami), hadi_irawansyah@yahoo.com (H. Irawansyah), mdhgamal@unri.ac.id (M. D. H. Gamal)

\section{To cite this article:}

Bustami, Hadi Irawansyah, M. D. H. Gamal. Holt-Winters Forecasting Method That Takes into Account the Effect of Eid. Science Journal of Applied Mathematics and Statistics. Vol. 3, No. 6, 2015, pp. 257-262. doi: 10.11648/j.sjams.20150306.15

\begin{abstract}
This paper discusses the Holt-Winters forecasting method that takes into account the effect of Eid. This method is used to predict the total domestic passengers departing in five major ports in Indonesia. Then a comparison is carried out between Holt-Winters method and Holt-Winters method that takes into account the effect of Eid. The comparison is done by comparing the mean square error obtained by both methods of forecasting, and it shows that the modified method provides better forcasting results.
\end{abstract}

Keywords: Forecasting, Holt-Winters Method, Effect of Eid, Mean Square Error

\section{Introduction}

Forecasting is a projection of one or more future events based on past data and current data [7, p.1]. Forecasting is needed to predict what will happen in the future. Data which will be forecasted can be any data that depend on the time series, including data affected by the holiday. Eid, the greatest religious holiday celebrated by Muslims worldwide, is not fixed to the Gregorian calendar, but it moves every year. This makes forecasting time series data a little bit difficult becauce the forecasting only records the seasonal influences for events where the days on which it occurs are fixed.

Many forecasting methods considering the calender variation have been proposed in the literatures. Bell and Hillmer [1] use ARIMA models for autocorrelation, trends, and seasonality, while for trading day variation and Easter holiday variation they use the regressian-type models. Jin and Tian [3] apply the Bell and Hillmer's method to analyze the important Chinese holidays in Taiwan, that might be affected by Chinese lunar calender that moves between two solar months. Consumption, production, and other economics behavior in Taiwan are strongly affected by these holidays. Monsell [6] suggests several alternative approaches to modify Easter holiday effects. These approaches include adaptation of Easter regressor (see Zhang et al. [10]), and the models that split the Easter model into separate weekday and weekend effects.

In this paper, Holt-Winters forecasting method is modified to capture the effect of Eid. The modification is carried out for the seasonal factors of the months affected by Eid. The modification of the seasonal factors is a combination of weighted effects of Eid for each month and the seasonal factors for each month. Weighted effects of Eid follow Shuja et al. [9]. In this article the forecasting and the effect of Eid are explained, then Holt-Winters forecasting method is modified by paying attention to the effects of Eid holiday, where the exodus occurs by the time of Eid celebration. Then, the modification of Holt-Winters method is tested to the data of total domestic passengers departing in five major ports in Indonesia. Then it ends up with a conclusion.

\section{Forecasting and the Effect of Eid}

Forecasting uses the time series data, which is a collection of observations conducted at any time from one or several variables being observed [2, p.1014], while Makridakis et al. $[5$, p.21] states that the time series data is the historical data of the observed, recorded any given time. Time series data patterns that can be observed may be constant, seasonal, cyclical, and/or trend.

Holt-Winters method is a method of conducting quantitative forecasting that approaches seasonal and trends. This method was first introduced by Charles C. Holt in 1957 and continued by his student Peter Winters in 1960 [4, p.5]. Holt-Winters method is divided into two methods: HoltWinters additive method and Holt-Winters multiplicative method.

In Holt-Winters method, time series data are grouped into two sections: the initial data and testing data. The initial data are the data of two seasonal periods or the first two years of time series data. The initial data are used to obtain the initial 
value of the base level, trends and seasonal factors. While the test data are used to make the process of forecasting with the number of data $p$. In the test data, the value of the base level, trend, and seasonal factors are calculated for each period.

Initial base level is given by the equation

$$
L_{0}=\frac{1}{h} \sum_{i=1}^{h} X_{i}
$$

where $L_{0}$ is an initial base level, $h$ is the amount of initial data and $X_{1}, X_{2}, X_{3}, \ldots, X_{h}$ are the initial data. Initial trend is given by the equation

$$
T_{0}=\frac{\bar{X}_{t 2}-\bar{X}_{t 1}}{m}
$$

where $T_{0}$ is the initial trend, $\bar{X}_{t 1}$ is the average of the initial first year data, $\bar{X}_{t 2}$ is the average of the second year data and $m$ is the number of seasons used in forecasting.

The number of seasons used is $m$ seasons, then there are $m$ initial seasonal factors. If we use Holt-Winters additive method, the initial seasonal factors are given by equation

$$
S_{i}=\bar{X}_{m-i}-L_{0}
$$

where $i=0,1,2, \ldots, m-1, \bar{X}_{m}$ is the average of the initial data for season $m, \bar{X}_{m-1}$ is the average initial data for season $(m-1)$, and so on.

If we use Holt-Winters multiplicative method, the initial seasonal factorsare given by equation

$$
S_{i}=\frac{\bar{X}_{m-i}}{L_{0}}
$$

where $i=0,1,2, \ldots, m-1, \bar{X}_{m}$ is the average of the initial data for the season $m, \bar{X}_{m-1}$ is the average initial data for season $(m-1)$, and so on.

Holt-Winters additive method is used for data containing trend and seasonal fluctuating additively. To forecast the next $k$ data period at the end of period $n$, the following equations are used:

$$
F_{n+k}=L_{n}+k T_{n}+S_{n+m-k}
$$

where $F_{n+k}$ is the forecasting of $k$ data periods based on data period $n, L_{n}$ is the base level for period $n, T_{n}$ is the trend for period $n$, and $S_{n-m+k}$ is the seasonal factors for period $(n-m+k)$. To get $L_{n}, T_{n}$ and $S_{n}$, the following formulae are used:

a) Base level equations are computed using the following formula:

$$
L_{n}=\alpha\left(X_{n}-S_{n-m}\right)+(1-\alpha)\left(L_{n-1}+T_{n-1}\right)
$$

where $\alpha$ is the smoothing parameter for the base level, $X_{n}$ is data for period $n, S_{n-m}$ is a seasonal factor for period $(n-m), L_{n-1}$ is the base level for period $(n-1)$ and $T_{n-1}$ is trend for period $(n-1)$.

b) Trend equations are evaluated using the following formula:

$$
T_{n}=\beta\left(L_{n}-L_{n-1}\right)+(1-\beta) T_{n-1}
$$

where $\beta$ is the smoothing parameter for the trend.

c) Seasonal factor equation computed using the following formula:

$$
S_{n}=\gamma\left(X_{n}-L_{n}\right)+(1-\gamma) S_{n-m}
$$

where $\gamma$ is the smoothing parameter for the seasonal factor.

Holt-Winters multiplicative method is used to data containing trend and seasonal fluctuating multiplicatively. To forecast the next $k$ data period at the end of period $n$, the following formulae are used:

$$
F_{n+k}=\left(L_{n}+k T_{n}\right) S_{n+m-k}
$$

where the smoothing equations are as follows:

a) Base level equations

$$
L_{n}=\alpha\left(\frac{X_{n}}{S_{n-m}}\right)+(1-\alpha)\left(L_{n-1}+T_{n-1}\right)
$$

b) Trend equations

$$
T_{n}=\beta\left(L_{n}-L_{n-1}\right)+(1-\beta) T_{n-1}
$$

c) Seasonal factor equations

$$
S_{n}=\gamma\left(\frac{X_{n}}{L_{n}}\right)+(1-\gamma) S_{n-m}
$$

A good forecasting is the one that generates small forecasting errors. Forecasting error indicators used in this article are the Mean Square Error (MSE). The MSE is obtained from the equation

$$
M S E=\frac{1}{p} \sum_{n=1}^{p}\left(X_{n}-F_{n}\right)^{2}
$$

where $p$ is the number of test data, $X_{n}$ is the data of period $n$ and $F_{n}$ is forecasting for the data of period $n$.

Furthermore, the optimal smoothing parameters $\alpha, \beta$, and $\gamma$ can be determined by referring to Rasmussen [8]. Rasmussen modeling method of forecasting is a linear programming model with the goal is to minimize the forecasting errors. The linear programming problem is solved with the help of solver on Microsoft Excel software.

Effect of Eid

Effect of Eid is the effect caused by the celebration of Eid. Shuja' et al. [9] uses the number of days affected by the celebration of Eid to determine the effect of Eid. In this article it is assumed that the number of days affected by the celebration of Eid is as long as 14 days, that is 7 days before and 7 days after the Eid holidays. Define $w$ as the total number of days affected by the celebration of Eid. Further define $\omega$ as the weight value of the effect of Eid. There are 
two cases to consider the $\omega$, based on the date of the celebration of Eid occured.

Case1.

If Eid falls between $1^{\text {st }}$ to $15^{\text {th }}$ of the month, then the effect will affect the month of Eid and the month before the month of Eid. Define the weight by the number of days affected by Eid for each month, that is

$$
\omega= \begin{cases}\frac{w_{l}}{w} & , \text { for month of Eid } \\ \frac{w_{l-1}}{w} & , \text { for month before month of Eid }\end{cases}
$$

where $w_{l}$ is the number of days affected in the month of Eid, $w_{l-1}$ is the number of days affected in the month before the month of Eid and $w$ is total number of days affected by the celebration of Eid. The relation among $w_{l}, w_{l-1}$ and $w$ can be written as follows:

$$
w=w_{l}+w_{l-1}
$$

For example, suppose Eid falls in October 3, then this is the case 1. Month $l$ is October and month $(l-1)$ is September. The value of $w_{l}=9, w_{l-1}=5$ and $w=14$, so that the weight values for October and September become

$$
\omega= \begin{cases}\frac{9}{14} & , \text { for October } \\ \frac{5}{14} & , \text { for September }\end{cases}
$$

Illustration how to determines $w_{l}$ and $w_{l-1}$.can be seen in Figure 1.

\begin{tabular}{|l|c|c|c|c|c|c|c|c|c|c|c|c|c|c|}
\hline \multicolumn{3}{|c|}{ September } & 1 & 2 & 3 & 4 & 5 & 6 & 7 & 8 & 9 & 10 \\
\hline 26 & 27 & 28 & 29 & 30 & \multicolumn{8}{|c|}{ October } \\
\hline
\end{tabular}

Figure 1. Illustration to Determine $w_{l-1}$ and $w_{l}$.

Case 2.

Case 2 happen if Eid falls between $16^{\text {th }}$ to $31^{\text {st }}$. The effect of Eid will influence the month of Eid and the month after the month of Eid. Define the weight by the number of days affected by Eid for each month, that is

$$
\omega=\left\{\begin{array}{l}
\frac{w_{l}}{W}, \text { for month of Eid } \\
\frac{w_{l-1}}{W}, \text { for month after month of Eid }
\end{array}\right.
$$

where $w_{l}$ is the number of days affected in the month of Eid, $w_{l+1}$ is the number of days affected in the month after the month of Eid and $W$ is the total number of days affected by Eid celebration. The relations among $w_{l}, w_{l+1}$ and $W$ can be written as follows:

$$
W=w_{l}+w_{l+1}
$$

For example, suppose Eid falls in September 27th, then the case is case 2. Month $l$ is September and month $(l+1)$ is October. The values of $w_{l}=10, w_{l+1}=4$ and $W=14$, so that the weight values for the months of September and October become

$$
\omega=\left\{\begin{array}{l}
\frac{10}{14}, \text { for September } \\
\frac{4}{14}, \text { for October }
\end{array}\right.
$$

Illustration to determine $w_{l}$ and $w_{l+1}$ can be seen in Figure 2 .

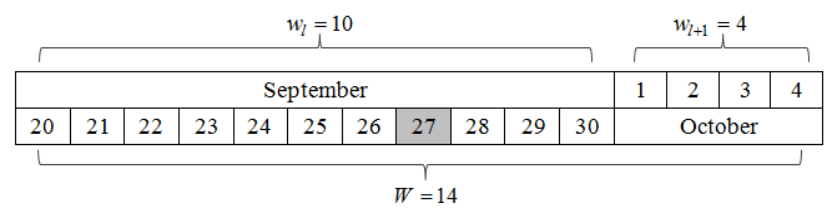

Figure 2. Illustration to Determine $w_{l}$ and $w_{l+1}$.

\section{Modification of Holt-winters Method}

Eid is one of the factors that affects the seasonal data, therefore incooperating the effects of Eid to the forecasting method of Holt-Winters influences the seasonal factor equations. The formula changes in determining the seasonal factors for Holt-Winters method due to taking into account the effects of Eid. Changes in formula are divided into 2 cases as to determine the effect of the noticed date to celebrate the Eid holiday.

Case 1.

In the first case, there are effects of Eid in the month of Eid and the month before the months of Eid. Modified seasonal factor is a combination of seasonal factors affected by Eid, so

$$
S_{\text {modification }}=\omega S_{l}+(1-\omega) S_{l-1}
$$

where $S_{l}$ is a seasonal factor in Eid, $S_{l-1}$ is a seasonal factor in the month before the month of Eid and $\omega$ is the weight of Eid effects.

Weights from equations (14) are adjusted to each month so that equation (18) becomes

$$
\begin{gathered}
S_{\text {Eid }}=\frac{w_{l}}{w} S_{l}+\left(1-\frac{w_{l}}{w}\right) S_{l-1} \\
S_{\text {Eid-1 }}=\frac{w_{l-1}}{w} S_{l}+\left(1-\frac{w_{l-1}}{w}\right) S_{l-1}
\end{gathered}
$$

The relations among $w_{l}, w_{l-1}$ and $w$ as being indicated by equation (15) cause equation (19) and equation (20) have another form that can be rewritten as

$$
S_{\text {Eid }}=\frac{w_{l}}{w} S_{l}+\frac{w_{l-1}}{w} S_{l-1}
$$




$$
S_{\mathrm{Eid}-1}=\frac{w_{l-1}}{w} S_{l}+\frac{w_{l}}{w} S_{l-1}
$$

Case 2.

The similar way as case 1 is applied to case 2 , the difference is forthe monthsaffected by the Eid celebration. In the case 2 Eid influences month $l$ and month $(l+1)$. Modifications for case 2 follows the form of equation (18) so that

$$
S_{\text {modification }}=\omega S_{l}+(1-\omega) S_{l+1}
$$

where $S_{l}$ is a seasonal factor in Eid, $S_{l+1}$ is a seasonal factor to the months after the month of Eid and $\omega$ is the weight of Eid effects.

Weights from equation (16) are adjusted to each month so that equation (23) becomes

$$
\begin{gathered}
S_{\text {Eid }}=\frac{w_{l}}{W} S_{l}+\left(1-\frac{w_{l}}{W}\right) S_{l+1} \\
S_{\text {Eid+1 }}=\frac{w_{l+1}}{W} S_{l}+\left(1-\frac{w_{l+1}}{W}\right) S_{l+1} .
\end{gathered}
$$

The relations among $W, w_{l}$ and $w_{l+1}$ as shown by equation (17) cause equations (24) and (25) change to

$$
\begin{gathered}
S_{\text {Eid }}=\frac{w_{l}}{W} S_{l}+\frac{w_{l+1}}{W} S_{l+1} \\
S_{\text {Eid }+1}=\frac{w_{l+1}}{W} S_{l}+\frac{w_{l+1}}{W} S_{l+1} .
\end{gathered}
$$

\section{Implementation of Method}

Holt-Winters modification method is tested on data of total domestic passangers departing from five major ports in Indonesia from year 2006 to 2014. Each data set from five major ports in Indonesia is tested using the method. In this paper, however, the detailed explanation of this modified Holt-Winters method only uses the data from port of Tanjung Priok, Jakarta. Then to see the data pattern, the data from port of Tanjung Priok are plotted as seen in Figure 3.

Figure 3 shows that there is an increase and a decrease in the data and they happen repeatedly, indicating that there are seasonal patterns in the data. In addition, from Figure 3, it can be seen that the trend of departing domestic cruise passengers shows a decrease in number every year.

It is known that from year 2006 to 2014 Eid coincides in August, September, October and November, therefore the data of departure for months of August to November are plotted as shown in Figure 4. This is done to see the effect of Eid in the data.

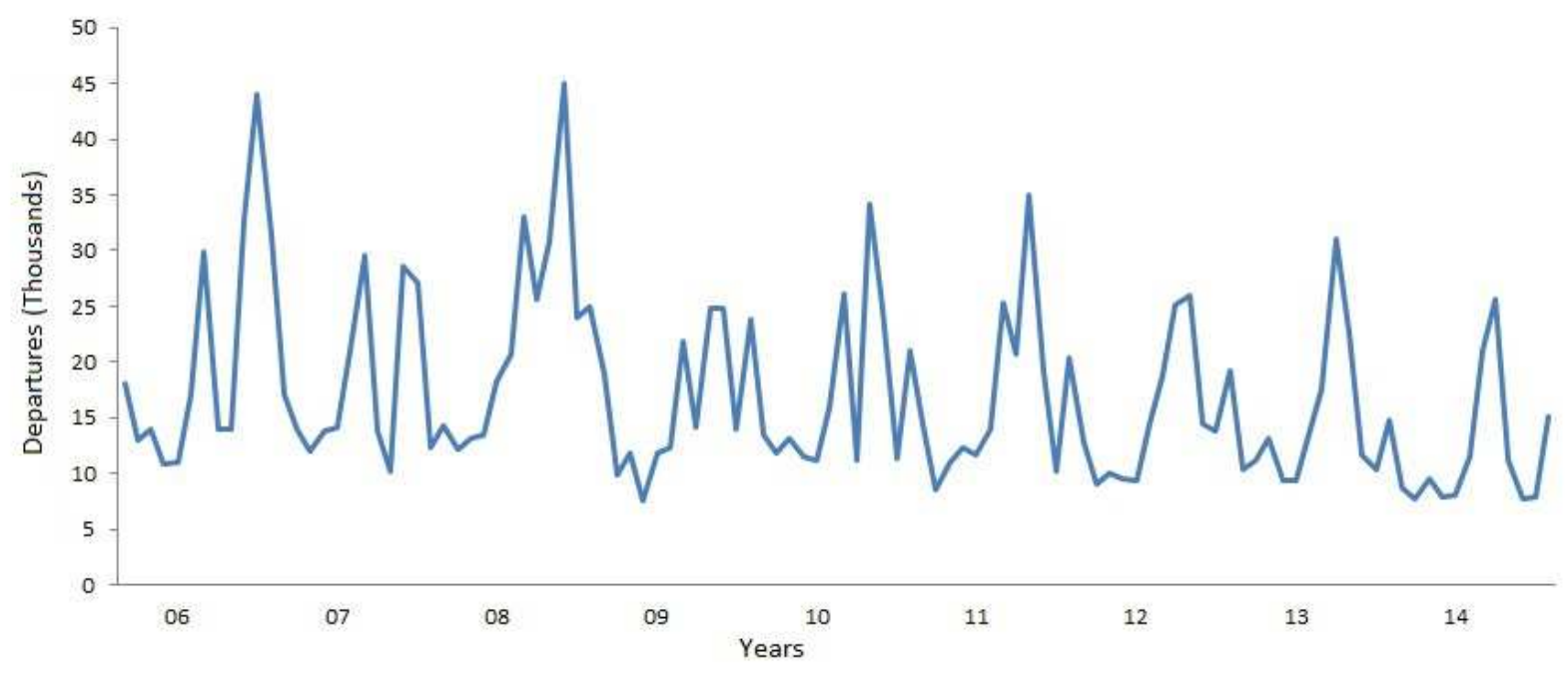

Figure 3. Plot Data in the Port of Tanjung Priok.

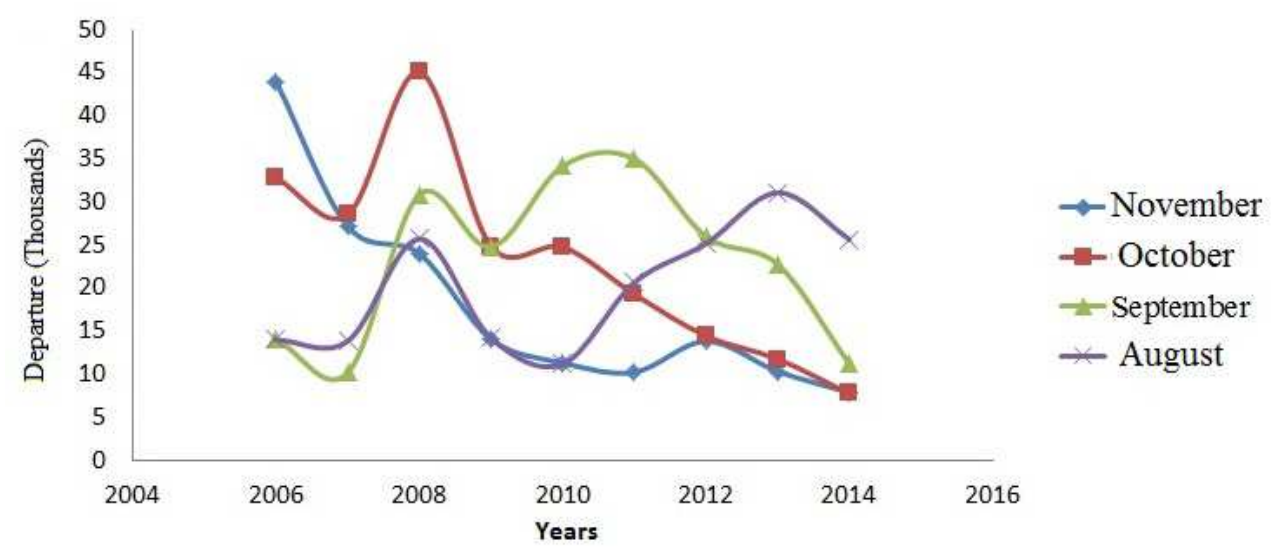

Figure 4. Plot Data for August, September, October and November. 


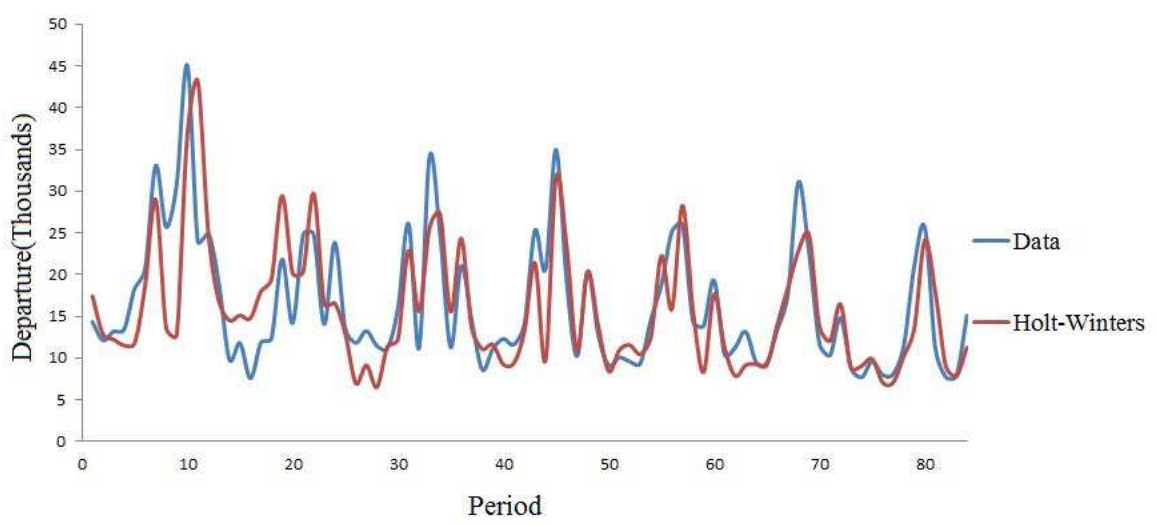

Figure 5. Data vs Forecasting with Holt-Winters Method.

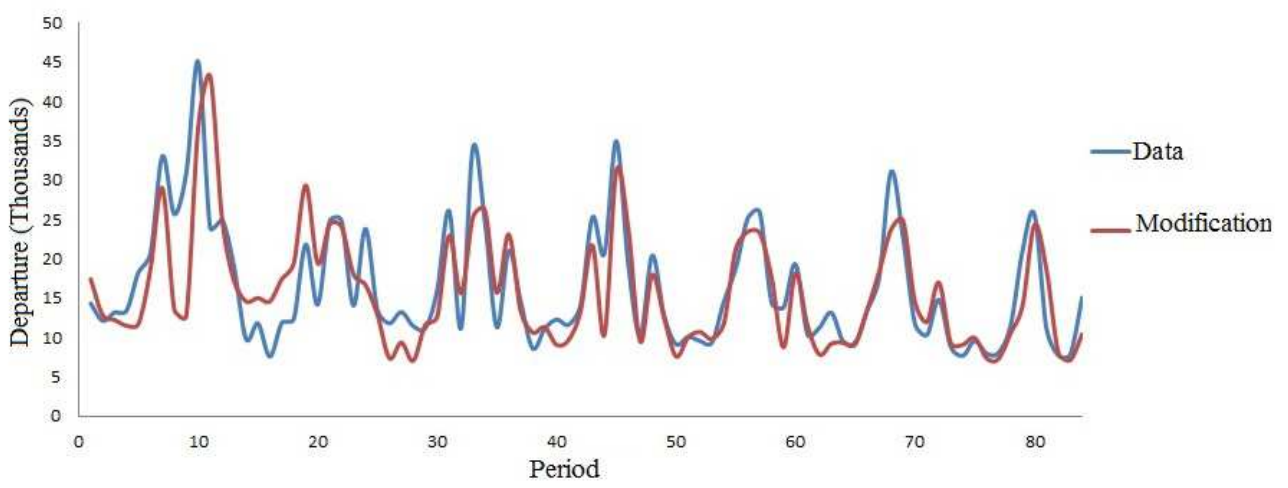

Figure 6. Data vs Forecasting with Modification Method.

In Figure 4, it can be seen that the Eid effects affect the data, therefore the modified Holt-Winters method can be tested on the data. The first thing to perform in implementing the Holt-Winters method is to see the seasonal data pattern. The departure data in Tanjung Priok fluctuate very extreme about the average so that the appropriate method to use is the Holt-Winters multiplicative method.

By using Holt-Winters the MSE value of 25.692 is obtained with optimal parameters $\alpha$ is $0.105, \beta$ is 0.001 and $\gamma$ is 0.982 . Plot data of departure passengers if compared to the results of the Holt-Winters forecasting method can be seen in Figure 5.

Forecasting results for year 2015 are used equation (9) where $k=1$ to forecast in January to $k=12$ for the forecast in December. Forecasting results for year 2015 are presented in Table 1.

Table 1. Forecasting Result with Holt-Winters Method.

\begin{tabular}{ll}
\hline Month & Forecasting \\
\hline January 2015 & 7,036 \\
February 2015 & 6,273 \\
March 2015 & 7,730 \\
April 2015 & 6,245 \\
May 2015 & 6,152 \\
June 2015 & 8,548 \\
July 2015 & 14,329 \\
August 2015 & 17,410 \\
September 2015 & 8,176 \\
October 2015 & 5,620 \\
November 2015 & 5,640 \\
December 2015 & 10,111 \\
\hline
\end{tabular}

Next, the forecasting results obtained from Holt-Winters method are modified. The modification is carried out with the hope that the value of the indicator of forecasting errors generated becomes smaller. Using a modified method of Holt-Winters we obtain the MSE equal to 23.770 with the optimal parameters $\alpha=0,105, \beta=0.001$, and $\gamma=0.982$. Departure plot data when compared to the forecasting results of the modified Holt-Winters can be seen in Figure 6 .

Forecasting results of total passengers that depart for year 2015 from the port of Tanjung Priok can be seen in Table 2 .

Table 2. Forecasting Results with Modification Method.

\begin{tabular}{ll}
\hline Month & Forecasting \\
\hline January 2015 & 6,488 \\
February 2015 & 5,748 \\
March 2015 & 7,174 \\
April 2015 & 5,913 \\
May 2015 & 5,901 \\
June 2015 & 8,274 \\
July 2015 & 17,178 \\
August 2015 & 18,639 \\
September 2015 & 9,760 \\
October 2015 & 6,281 \\
November 2015 & 6,204 \\
December 2015 & 11,011 \\
\hline
\end{tabular}

In addition to the data in the port of Tanjung Priok, modified Holt-Winters method is also tested to data in port of Belawan, Tanjung Perak, Makassar and Balikpapan. MSE generated by Holt-Winters method and Holt-Winters modification method for the data of each port can be seen in Table 3 . 
Table 3. A Comparison between MSE Holt-Winters and Modification.

\begin{tabular}{lll}
\hline Port & MSE Holt-Winters & MSE Modification \\
\hline Tanjung Priok & 25.691858 & 23.769693 \\
Belawan & 8.355402 & 7.117931 \\
Tanjung Perak & 247.386746 & 226.389962 \\
Makassar & 147.701650 & 147.182863 \\
Balikpapan & 77.712730 & 77.819097 \\
\hline
\end{tabular}

\section{Conclusion}

By modifying the Holt-Winters methods we obtained a forecasting method that can recognize well the effect of Eid. We have shown that the MSE generated by the modified HoltWinters method are smaller than the MSE produced by HoltWinters methods for the data of departure passengers in four different ports in Indonesia namely Belawan, Tanjung Priok, Tanjung Perak, and Makassar. However, for the forecasting departure data in Balikpapan, it is better to use the Holt-Winters forecasting method, since it provides $M S E$ smaller value than the one found by the modified Holt-Winters method.

\section{References}

[1] Bell, W. R. and S. C. Hillmer. (2003). Modeling Time Series with Calender Variation. Journal of the American Statistical Association, 78, pp. 526-534.

[2] Hillier, F. S. and G. J. Lieberman. (2001). Introduction to Operations Research, $7^{\text {th }}$ Ed. McGraw-Hill Higher Education. New York.
[3] Jin, L. L. and S. L. Tian. (2003). Modeling Lunar Calender Holiday Effect in Taiwan. Taiwan Economics Policy and Forecast, 33, pp 1-37.

[4] Hyndman, R. J., A. B. Koehler, J. K Ord, and R. D. Snyder. (2008). Forecasting with Exponential Smoothing-The State Approach. Springer-Verlag, Berlin Heidelberg.

[5] Markidakis, S., S. C. Wheelwright, and V. E. McGee. (1998). Forecasting: Methods and Applications, 3rdEd. Jhon Wiley \& Sons. New York.

[6] Monsell, B. C. (2007). Issues in Modeling and Adjusting Calender Effects in Economics Time Series. Paper presented at the ICES-III, June 18-21, 2007, Montreal, Quebec, Canada.

[7] Montgomery, D. C., C. L. Jenning, and M. Kulahci. (2008). Introduction to Time Series Analysis and Forecasting. WileyInterscience. New Jersey.

[8] Rasmussen, R. (2003). On Time Series Data and Optimal Parameters. Omega, 32, pp. 111-120.

[9] Shuja', N., M. A. Lazim, and Y. B. Wah. (2007). Moving Holiday Effects Adjustment for Malaysian Economic Time Series. Journal of Department of Statistics Malaysia, 1, pp. $35-50$.

[10] Zhang, X., C. H. McLaren, and C. C. S. Leung. (2003). An Easter Proximity Effect: Modeling and Adjusment. Australian and New Zealand Journal of Statistics, 43, pp. 269-280. 